\title{
Ollier disease
}

INSERM

\section{Source}

INSERM. (1999). Orphanet: an online rare disease and orphan drug data base. Ollier disease. ORPHA:296

Enchondromatosis is a rare primary bone dysplasia disorder characterized by the development of multiple mainly unilateral or asymmetrically distributed enchondromas throughout the metaphyses of the long bones. 